\title{
SISTEMATIZACIÓN DE PRÁCTICAS REHABILITACIÓN EN TIEMPOS DE PANDEMIA: SÍNDROME POST UNIDAD DE CUIDADOS INTENSIVOS. (UN SÍNDROME QUE REQUIERE ATENCIÓN).
}

\author{
REHABILITATION IN TIMES OF PANDEMIC: POST-INTENSIVE CARE SYNDROME. (A \\ SYNDROME THAT REQUIRES ATTENTION).
}

\section{Fabrizio Acevedo P. ${ }^{1}$, Felipe Ceballos V. ${ }^{2}$, Maria Paz Guzmán S. ${ }^{3}$}

\begin{abstract}
RESUMEN
La epidemia de COVID-19 ha llevado a la necesidad de decisiones sin precedentes. Esta sistematización aborda la toma de decisiones operativas que el equipo de Terapia Ocupacional del Hospital estatal San José de Santiago ha trabajado durante la epidemia
\end{abstract}

PALABRAS CLAVE

Terapia Ocupacional, Cuidados intensivos, pandemia COVID-19.

\begin{abstract}
The COVID-19 epidemic has led to the need for unprecedented decisions. This systematization addresses the operational decision-making that the Occupational Therapy team of the San José de Santiago State Hospital has worked on during the epidemic.
\end{abstract}

\section{KEYWORDS}

Occupational Therapy, Intensive Care, COVID-19 Pandemic

Recibido: $22 / 07 / 2020$

Aceptado: $25 / 08 / 2020$

1 Terapeuta Ocupacional, Mg® Neurociencias, Universidad de Chile. Unidad de Geriatría/Medicina Física y Rehabilitación, Hospital Clínico San José, Santiago, Chile Docente Escuela Terapia Ocupacional Universidad de Santiago de Chile y Universidad Nacional Andrés Bello. flacevedo@uchile.cl +56962463187

2 Terapeuta Ocupacional Licenciado en Ciencias de la Ocupación Humana, Universidad de Chile. Unidad de Geriatría/Medicina Física y Rehabilitación, Hospital Clínico San José, Santiago, Chile. ceballos.to@gmail.com +56985854847

3 Terapeuta Ocupacional Licenciado en Ciencias de la Ocupación Humana, Universidad de Chile. Unidad de Geriatría/Medicina Física y Rehabilitación, Hospital Clínico San José, Santiago, Chile pazigs@gmail.com +56994370568 
$\mathrm{P}$ reocupación ha causado la situación actual de pandemia por coronavirus (CoviD-19) a nivel país, provocando cambios en los distintos servicios hospitalarios de la red pública de salud, con énfasis en los equipos que se desempeñan en unidades críticas. Según datos obtenidos en un estudio chileno realizado por Ruiz et al. (2016) que describe la epidemiología general de personas en una unidad crítica de un hospital público, la estadía en una unidad de cuidados intensivos (UCI) y el soporte de ventilación mecánica tienen una mediana de 5 y 3 días respectivamente, siendo los motivos médicos de ingreso más frecuentes; el neurocrítico, la sepsis severa y la insuficiencia respiratoria. Datos que se han visto modificados durante la pandemia por CoviD-19 asociado a dificultad respiratoria aguda, sepsis y shock séptico, o falla multiorgánica, incluyendo lesiones renales, hepáticas y cardíacas, que generalmente requerirán ventilación mecánica invasiva en camas de unidades críticas, repercutiendo en el tiempo promedio de estadía, siendo de 8 días (OPS, 2020), lo cual ha emplazado la suspensión de cirugías y procedimientos electivos y no urgentes por el aumento de personas hospitalizadas por CovID-19, reconfigurando las camas medicas/ quirúrgicas a camas críticas con la finalidad de cubrir la demanda hospitalaria.

Considerando lo anterior, la presencia del Covid-19 no solo ha impactado en la salud pública del país a nivel asistencial, sino también por su gravedad, asociada a mayor necesidad de cuidados intensivos, repercutiendo en los equipos de rehabilitación, quienes han puesto sus mayores esfuerzos tanto en la prevención de trastornos y síndromes disfuncionales asociados al contexto de unidades críticas, como también en su manejo y tratamiento especializado. Uno de ellos es el Síndrome post cuidados intensivos (SPCl), el cual se define como una alteración nueva o un empeoramiento en el estado físico (debilidad muscular esquelética y respiratoria) cognitivo (funciones ejecutivas, memoria y velocidad de procesamiento) o mental (ansiedad y estrés post traumático) que surge y persiste a largo plazo después de una hospitalización por una enfermedad crítica (Ohtake et al., 2018).

Una de las alteraciones más estudiadas recientemente como parte de los SPCI, es la debilidad adquirida en unidad de cuidados intensivos (DA-UCI), descrita como debilidad generalizada de las extremidades que se desarrolla mientras el paciente está gravemente enfermo, para lo cual no hay otra explicación que la enfermedad crítica en sí (Fan et al.,2014) y que generalmente se acompaña de debilidad diafragmática que dificulta el proceso de weaning o destete de la ventilación mecánica invasiva (Díaz et al., 2017). Lo anterior se asocia a duración prolongada de ventilación mecánica y estadía en UCl, rehabilitación tardía y mayor riesgo de morbilidad y mortalidad (Gosselink, Needham y Hermans, 2012). Actualmente, todos estos factores están presentes con mayor frecuencia en los hospitales, lo generando un aumento de la prevalencia del SPCl, y por ende de DA-UCI, siendo la rehabilitación temprana una herramienta fundamental para la prevención de este síndrome y aún más relevante para la disminución de la incidencia de DA-UCI (Fuke, 2018). Por lo tanto, tal como dicen las directrices de la Organización mundial de la salud (2020) en su documento manejo clínico de CoviD-19, se llama a las autoridades de salud a generar las facilidades para rehabilitación no solamente a nivel intrahospitalario, sino también en atención ambulatoria o virtual, considerando las implicancias a largo plazo del SPCI.

Actualmente las y los Terapeutas Ocupacionales que trabajan en hospitales del sistema de salud pública están enfrentando diariamente diversos desafíos en atención hospitalaria, tales como la prevención y manejo no farmacológico del delirium, confección y ejecución de protocolos de posicionamiento (especialmente en personas en posición decúbito prono), acompañamiento comprometido a los usuarios y familias en sus procesos de salud-enfermedad, cuidados de final de vida, telerehabilitación, entre otros. Sin embargo, dar repuesta a estos desafíos no solo depende de la intervención del Terapeuta Ocupacional como parte de un equipo rehabilitador, sino de la interrelación que existe entre los diferentes actores en el proceso de salud. Es por esto, que el objetivo de esta carta es describir las principales problemáticas y sugerentes soluciones del quehacer profesional como parte de un equipo interdisciplinario, que permita enfrentar las consecuencias que se han ido presentando en nuestra praxis.

Primero, no se cuenta con un registro nacional del número de personas que presentan DA-UCI (como se dijo previamente uno de los síndromes más prevalentes dentro de los SPCI). En los reportes REM del ministerio de salud año 2019 (MINSAL, 2020) no existe el diagnóstico específico de DA-UCI para registrarlo en la planilla de estadística de ingreso a un programa de rehabilitación integral. Por consiguiente, debe existir mayor 
diálogo entre los clínicos y las autoridades que designan nomenclatura diagnóstica, formas de registro y recolección de datos. Esto permitiría realizar estadísticas que pudiesen dar paso a políticas públicas preventivas y de tratamiento.

Segundo, no existen policlínicos protocolizados multidisciplinarios que realicen un seguimiento desde el alta hospitalaria a las personas con SPCI. En la actualidad existen programas en los servicios de medicina física y rehabilitación que abarcan atenciones de múltiples disciplinas a personas con discapacidad física y/o cognitiva, sin embargo no hay consensos nacionales respecto a la pesquisa, evaluación, números de sesiones y tipo tratamiento integral de estos usuarios. Lo que si está claro, es que las personas con SPCı presentan secuelas a largo plazo (OPS, 202O) que requieren manejo de especialidades, tales como Medicina Interna (intensivistas), Fisiatría, Kinesiología, Fonoaudiología y Terapia Ocupacional. Específicamente en esta última, se hacen necesarias las prestaciones a nivel funcional, favoreciendo autonomía e independencia de personas con esta problemática de salud. Por lo tanto, se evidencia la necesidad de una clínica ambulatoria para usuarios con este síndrome en particular, ya que un número creciente de personas que sobreviven a una enfermedad crítica, especialmente durante esta pandemia, requerirán atenciones eficientes y de calidad, con la necesidad urgente de abordar más a fondo las consecuencias a largo plazo de los cuidados intensivos para los sobrevivientes y sus familias (Inoue et al., 2020).

Por último, siendo no menor, no se cuenta con el suficiente personal para abarcar la problemática anterior. A partir de esto se requiere imperiosamente conocimiento y respaldo de autoridades locales de salud (directores de servicios, hospitales, unidades, entre otros) sobre rehabilitación temprana en unidades críticas y su posterior seguimiento al alta, incluyendo la telesalud y/o telerehabilitación, proporcionando a su vez el acceso futuro a una atención ambulatoria futura. Facilitar que las familias y cuidadores de personas con SPCI reciban recursos educativos e información para su autonomía, especialmente cuando se anticipan barreras para acceder al seguimiento de rehabilitación (OMS, 2020).

Los Terapeutas Ocupacionales tienen la posibilidad de participar en los procesos de salud desde la etapa aguda hasta la atención ambulatoria, tanto en aspectos clínicos como administrativos, pudiendo establecer criterios claros que definan lineamientos para un mejor manejo de la problemática. Para ello es fundamental el trabajo en equipo, el establecimiento de roles profesionales y deberes en relación a la valoración de riesgo de SPCI y $\mathrm{DA}-\mathrm{UCl}$, la rigurosidad en las evaluaciones respectivas para diagnosticar dichos síndromes y la organización para establecer mecanismos de acción que logren dar respuesta a la población involucrada a corto mediano y largo plazo.

En conclusión, es vital establecer medios de comunicación claros entre los profesionales de rehabilitación y las autoridades, facilitando el diálogo y discusión de las formas de registro de las personas que están en rehabilitación, permitiendo tomar medidas acordes a su demanda en incidencia y prevalencia, como lo es en este caso el notable aumento por COVID-19 de DA$\mathrm{UCl}$, y por ende de SPCI, observado en la praxis clínica. A su vez, se evidencia la necesidad de generar un flujo de atención desde las unidades críticas hasta la atención abierta (Figura 1) que sea transversal en el sistema público hospitalario. También, la vinculación con la red familiar es fundamental, incluyéndolos de forma activa en el proceso, entregándoles información clara y precisa, la cual requiere experiencia y conocimiento de los profesionales implicados, individualizando las atenciones y pasos a seguir. Finalmente, se hace urgente emitir políticas públicas por parte del estado en torno a la discapacidad, como por ejemplo el acceso y planes de recuperación de este grupo durante y posterior a la pandemia. 
FIGURA 1. FLUJO DE ATENCIÓN USUARIO CON PSCI.

Ingreso a $\mathrm{UCl}$

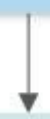

Intervención temprana en $\mathrm{UCl}$
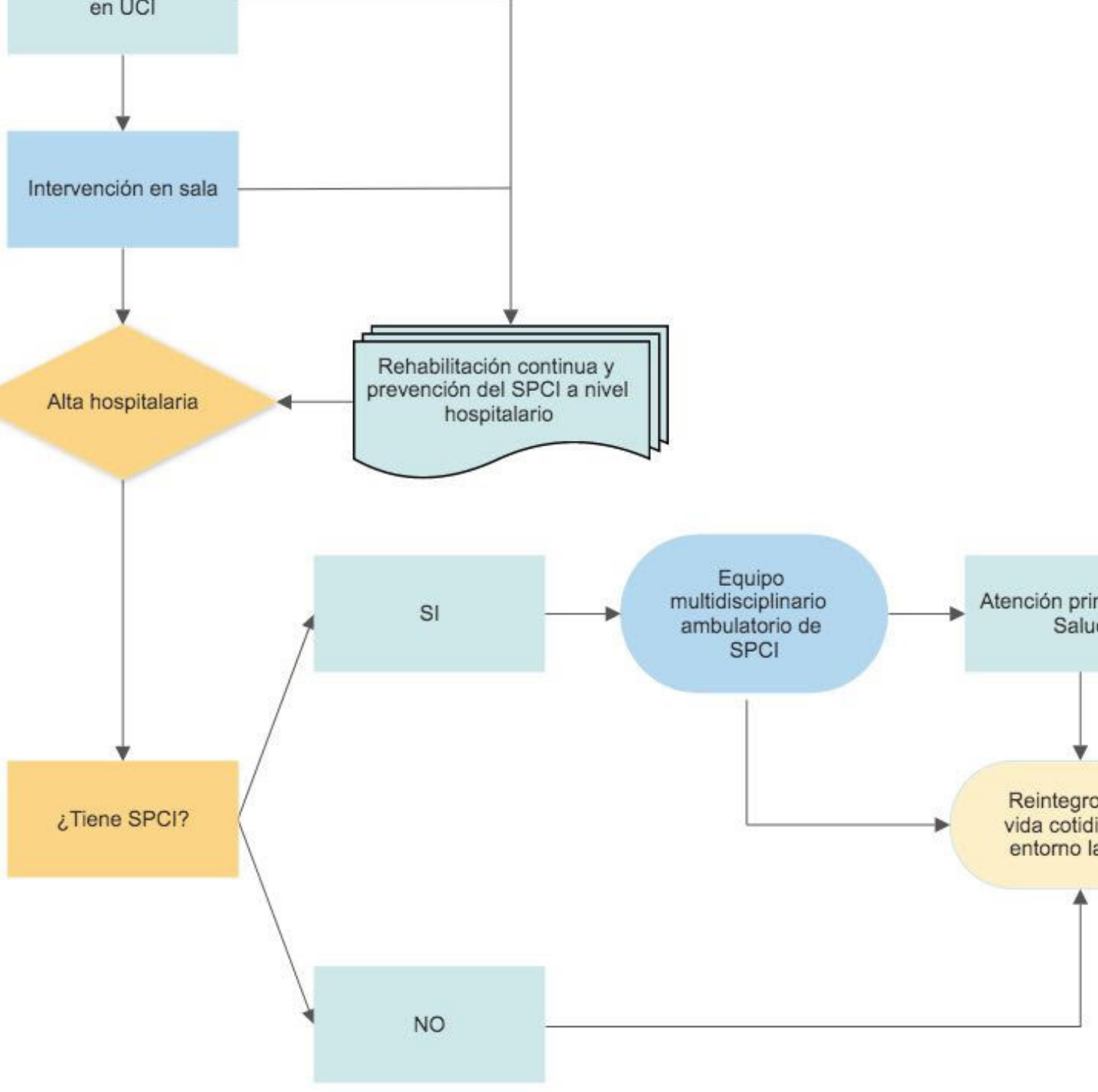


\section{ReFerenCiAS}

Diaz Ballve, Ladislao Pablo, Dargains, Nahuel, Urrutia Inchaustegui, Jose? García, Bratos, Antonella, Milagros Percaz, Maria de los, Bueno Ardariz, Cesar, Cagide, Sabrina, Balestrieri, Carolina, Gamarra, Claudio, Paz, Dario, Rotela, Eliana, Muller, Sebastian, Bustos, Fernando, Aranda Castro, Ricard, \& Settembrino, Esteban. (2017). Debilidad adquirida en la unidad de cuidados intensivos. Incidencia, factores de riesgo y su asociación con la debilidad inspiratoria. Estudio de cohorte observacional. Revista Brasileira de Terapia Intensiva, 29(4), 466-475. Epub December 07, 2017. https://dx.doi.org/10.5935/0103-507x.20170063.

Fan, E., Cheek, F., Chlan, L., Gosselink, R., Hart, N., Herridge, M. S., Hopkins, R. O., Hough, C. L., Kress, J. P., Latronico, N., Moss, M., Needham, D. M., Rich, M. M., Stevens, R. D., Wilson, K. C., Winkelman, C., Zochodne, D. W., Ali, N. A., ATS Committee on ICU-acquired Weakness in Adults, \& American Thoracic Society (2014). An official American Thoracic Society Clinical Practice guideline: the diagnosis of intensive care unit-acquired weakness in adults. American journal of respiratory and critical care medicine, 190(12), 1437-1446. https://doi.org/10.1164/rccm.201411-2011ST.

Fuke, R., Hifumi, T., Kondo, Y., Hatakeyama, J., Takei, T., Yamakawa, K., Inoue, S., \& Nishida, O. (2018). Early rehabilitation to prevent postintensive care syndrome in patients with critical illness: a systematic review and meta-analysis. BMJ open, 8(5), e019998. https://doi.org/10.1136/bmjopen-2017-019998.

Gosselink, R., Needham, D., \& Hermans, G. (2012). ICU-based rehabilitation and its appropriate metrics. Current opinion in critical care, 18(5), 533-539. https://doi.org/10.1097/MCC.0b013e328357f022.

Inoue, S., Hatakeyama, J., Kondo, Y., Hifumi, T., Sakuramoto, H., Kawasaki, T., Taito, S., Nakamura, K., Unoki, T., Kawai, Y., Kenmotsu, Y., Saito, M., Yamakawa, K., \& Nishida, O. (2019). Post-intensive care syndrome: its pathophysiology, prevention, and future directions. Acute medicine \& surgery, 6(3), 233-246. https:// doi.org/10.1002/ams2.415.

MINSAL (2020). NIVEL HOSPITALARIO. SECCIÓN B.1: INGRESOS Y EGRESOS AL PROGRAMA DE REHABILITACIÓN INTEGRAL. 15 Julio, 2020, de REPORTES REM Sitio web: https://reportesrem.minsal. $\mathrm{cl}$ /?_token=j2Ey7QtSkWmXfwPQOnxloOiUV0tlygjLuUf1DWuz \&serie $=1 \&$ rem $=74 \&$ seccion_id $=832 \&$ tipo $=3^{\circledR}$ iones $=0 \&$ servicios $=-$ $1 \&$ periodo $=2019 \&$ mes_inicio $=1 \&$ mes_final $=12$.

Ohtake, P. J., Lee, A. C., Scott, J. C., Hinman, R. S., Ali, N. A., Hinkson, C. R., Needham, D. M., Shutter, L., Smith-Gabai, H., Spires, M. C., Thiele, A., Wiencek, C., \& Smith, J. M. (2018). Physical Impairments Associated With Post-Intensive Care Syndrome: Systematic Review Based on the World Health Organization's International Classification of Functioning, Disability and Health Framework. Physical therapy, 98(8), 631-645. https://doi.org/10.1093/ptj/pzy059
OPS. (2020). Rehabilitation considerations during the COVID-19 outbreak. Julio 14, 2020, de OPS Sitio web: https://iris.paho. org/handle/10665.2/52035.

Ruiz, Carolina, Díaz, Miguel Ángel, Zapata, Juan Marcelo, Bravo, Sebastián, Panay, Sergio, Escobar, Cristina, Godoy, Jorge, Andresen, Max, \& Castro, Ricardo. (2016). Características y evolución de los pacientes que ingresan a una Unidad de Cuidados Intensivos de un hospital público. Revista médica de Chile, 144(10), 12971304. https://dx.doi.org/10.4067/S0034-98872016001000009.

World Health Organization. (2020). Clinical management of COVID-19: interim guidance. Recuperado de: https://www.who. int/publications/i/item/clinical-management-of-covid-19. 
